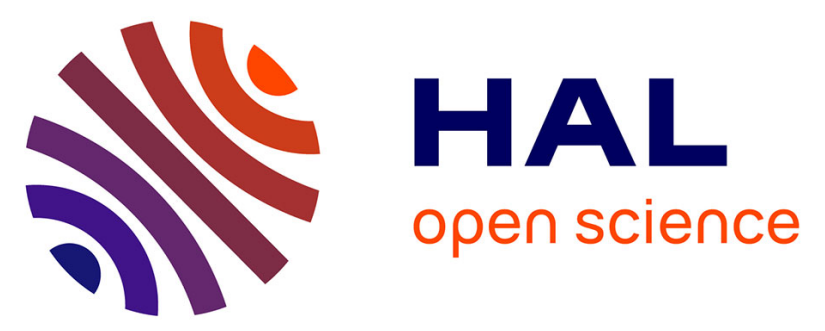

\title{
Dynamics and wettability of petroleum fluids in shale oil probed by 2D T1 -T2 and fast field cycling NMR relaxation
}

\author{
Jean-Pierre Korb, B. Nicot, I. Jolivet
}

\section{To cite this version:}

Jean-Pierre Korb, B. Nicot, I. Jolivet. Dynamics and wettability of petroleum fluids in shale oil probed by 2D T1 -T2 and fast field cycling NMR relaxation. Microporous and Mesoporous Materials, 2017, 10.1016/j.micromeso.2017.05.055 . hal-01539764

\section{HAL Id: hal-01539764 https://hal.sorbonne-universite.fr/hal-01539764}

Submitted on 15 Jun 2017

HAL is a multi-disciplinary open access archive for the deposit and dissemination of scientific research documents, whether they are published or not. The documents may come from teaching and research institutions in France or abroad, or from public or private research centers.
L'archive ouverte pluridisciplinaire HAL, est destinée au dépôt et à la diffusion de documents scientifiques de niveau recherche, publiés ou non, émanant des établissements d'enseignement et de recherche français ou étrangers, des laboratoires publics ou privés. 


\title{
Dynamics and wettability of petroleum fluids in shale oil probed by $2 D \mathrm{~T}_{1}-\mathrm{T}_{2}$ and fast field cycling NMR relaxation

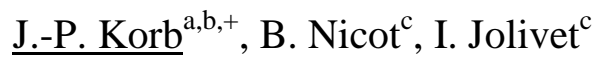

${ }^{a}$ Physique de la Matière Condensée, Ecole Polytechnique-CNRS, 91128 Palaiseau, France.

${ }^{\mathrm{b}}$ Sorbonne Université, UPMC Univ. Paris 06, Laboratoire Physicochimie des Electrolytes, Nanosystèmes Interfaciaux (Phenix), Paris Cedex 5, France.

${ }^{\mathbf{c}}$ Total EP, Centre Scientifique et Technique Jean Feger (CSTJF), 64018 Pau, France.

\begin{abstract}
We present nuclear magnetic relaxation dispersion of longitudinal relaxation rates $1 / T_{1}$ (NMRD) and 2D spin-correlation $T_{1}-T_{2}$ at different frequencies for oil and brine confined in shale oil rocks. We describe the nuclear spin relaxation models used for obtaining important dynamical and structural parameters from these experiments. These models allow interpreting the very different $T_{1} / T_{2}$ ratio observed for these petroleum fluids on the 2D spin-correlation $T_{1}-T_{2}$ observed at two frequencies. They give also new informations on the dynamics and wettability of the embedded fluids as well as some structural measurement on the kerogen pore size.
\end{abstract}

Keywords: Shale oil rocks, FFC relaxometry, oil and brine dynamics, wettability

${ }^{+}$Corresponding author

E-mail address: jean-pierre.korb@ polytechnique.fr

Tel: 33169334739 
It is of primary importance to probe in situ and non-invasively the dynamics and wettability of oil, water and gas trapped in the complex microstructure of shale-oil rocks. The main reason is because liquid and gas hydrocarbons can be produced from these organic and mineral sedimentary rocks. However, most of the usual techniques cannot separate these fluids in the complex microstructure of shale rocks. Several attempts of one-dimensional (1D) and two-dimensional (2D) low-field ${ }^{1} \mathrm{H}$ nuclear magnetic resonance (NMR) techniques have proven useful for probing the fluid dynamics in mature organic-shale reservoir rocks [1-3]. 2D spin-correlation NMR technique at low fields $\left(T_{1}-T_{2}\right)$ has proven useful for separating fluids in confinement [4] and can be made down-hole. Recently, we have used this 2D NMR technique for probing the saturation of oil and brine in shale rocks [5]. However, it is not directly sensitive to the dynamics of the embedded liquids. We have also evidenced by fast-field cycling relaxometry strongly different magnetic-field dependence (NMRD) of the longitudinal nuclear-spin-lattice relaxation rate $1 / T_{1}$ of oil and and brine embedded in shales [5]. As this technique explores a large magnetic field range allowing changing the fluctuations to which the nuclear spin relaxation is sensitive, it has offered multiscale opportunities for characterizing the molecular dynamics and transport properties of fluids embedded in confined environments [6]. But some questions still remain for interpreting the striking differences in the NMRD profiles as well as the far different $T_{1} / T_{2}$ ratio in the $\left(T_{1}-T_{2}\right)$ data of oil and brine in shales. Here, we come back to the necessary theoretical models in $1 \mathrm{D}$ and 2D NMR data used at variable frequencies for obtaining some new dynamical and structural information on the mineral (clay) and organic (kerogen) microstructures.

\section{Materials and methods}

The rocks samples (oil/water/air and water/air) come from a field producing light oil and are supplied by Total EP, France. The sponge-like microstructure of kerogen has been characterized with FIB SEM technique and presents a fractal distribution of pore sizes with a huge surface area $[7,8]$. We show in Fig.1 FIB SEM micrograph of the microporosity of a shale oil rock sample evidencing the quasi 1D 
density of $\eta_{S}=4.50 \times 10^{19}$ paramagnetic species (mainly $\mathrm{Mn}^{2+}$ ions) per gram of shale-oil sample in a single environment [5]. The analysis has revealed a density of $\rho_{\text {Shale }} \sim 2.6 \mathrm{~g} / \mathrm{cm}^{3}$. We have deduced a surface density of paramagnetic relaxation sinks $\sigma_{s}=\left(\eta_{S} \rho_{\text {Shale }}\right)^{2 / 3}=2.39 \times 10^{13} \mathrm{Mn}^{2+} / \mathrm{cm}^{2}$ assuming a homogeneous repartition in the sample. This gives an average distance between relaxing sinks about $1 / \sqrt{ } \sigma_{s} \sim 2 \mathrm{~nm}$ on pore surfaces. NMRD data have been performed on oil/water/air shale rocks at room temperature with a fast-field cycling (FFC) spectrometer from Stelar s.r.l., Mede, Italy [5]. For separating the NMRD responses of confined oil and water, we have used a standard procedure in the petroleum industry [5]. Last, we used 2D NMR $T_{1}-T_{2}$ correlation experiments performed at $2.5 \mathrm{MHz}$ and $23 \mathrm{MHz}$ with an Oxford Instrument spectrometer on "as received" oil/water/air shale rock which presents two different peaks associated to confined oil and water (brine).

\section{Experimental results, data treatment and discussion on NMRD in shales}

We used a home-written "Matlab" program [5] following closely the Butler-Reeds-Dawson [9] and Venkataramanan et al algorithms [10] for analyzing the longitudinal magnetization decays ending to a bimodal distribution of $T_{1}$ for all the Larmor frequencies studied between $10 \mathrm{kHz}$ and $35 \mathrm{MHz}$. As shown in inset of Fig. 2, the two thin peaks allow building two different $T_{1}$-NMRD profiles assigned to oil and water [11]. We have proposed theoretical models for describing the longitudinal and transverse nuclear spin relaxation of a fluid embedded either in 2D [12-14] or 1D [15] pore geometries. Here, we just outline the essential features of these models for water and oil fluids in confinement.

\subsection{NMRD profile of water confined in lamellar clay minerals}

We assume the general biphasic fast exchange model where the exchange time between the protonwater transiently belonging to the surface and the bulk in pores is shorter than their respective relaxation times. When considering the lamellar clay mineral modeled as a 2D system, the main contribution of the proton relaxation comes from the $2 \mathrm{D}$ translational proton-water $(I)$ diffusion at proximity of the fixed 
paramagnetic relaxing sinks $\left(\mathrm{Mn}^{2+}\right)$ of $\operatorname{spins} S=5 / 2$ that modulates their relative dipole-dipole interaction.

The numerous 2D molecular reencounters $\left({ }^{1} \mathrm{H}-\mathrm{Mn}^{2+}\right)$ are responsible for the net frequency dependence observed in Fig. 2. In that case, we have previously found that the longitudinal and transverse relaxation times are given by [12-14]:

$$
\begin{aligned}
R_{1, \text { water }}\left(\omega_{I}\right)=R_{1, \text { bulk }}+\left[\pi /\left(30 \delta_{\text {water }}^{3}\right)\right] \sigma_{S} \rho_{\text {water }} S_{p, N M R}\left(\gamma_{I} \gamma_{S} \hbar\right)^{2} S(S+1) \tau_{m} \\
\times\left[3 \ln \left(\frac{1+\omega_{I}^{2} \tau_{m}^{2}}{\left(\tau_{m} / \tau_{s}\right)^{2}+\omega_{I}^{2} \tau_{m}^{2}}\right)+7 \ln \left(\frac{1+\omega_{S}^{2} \tau_{m}^{2}}{\left(\tau_{m} / \tau_{s}\right)^{2}+\omega_{S}^{2} \tau_{m}^{2}}\right)\right] \\
R_{2, \text { water }}\left(\omega_{I}\right)=R_{2, \text { bulk }}+\left[2 \pi /\left(30 \delta_{\text {water }}^{3}\right)\right] \sigma_{S} \rho_{\text {water }} S_{p, N M R}\left(\gamma_{I} \gamma_{S} \hbar\right)^{2} S(S+1) \tau_{m} \\
\times\left[2 \operatorname{Ln}\left(\frac{\tau_{S}}{\tau_{m}}\right)+\frac{3}{4} \ln \left(\frac{1+\omega_{I}^{2} \tau_{m}^{2}}{\left(\tau_{m} / \tau_{s}\right)^{2}+\omega_{I}^{2} \tau_{m}^{2}}\right)+\frac{13}{4} \ln \left(\frac{1+\omega_{S}^{2} \tau_{m}^{2}}{\left(\tau_{m} / \tau_{s}\right)^{2}+\omega_{S}^{2} \tau_{m}^{2}}\right)\right]
\end{aligned}
$$

In Eqs. 1 and 2, $\gamma_{I}$ and $\gamma_{S}=658 \gamma_{I}$ are the gyromagnetic ratio of the proton and electron, $\sigma_{S}$ is the surface density of paramagnetic sources of relaxation that we can probe by electron spin resonance [5] and $\rho_{\text {water }}$ is the water density. Here, we have considered a thin surface layer $\lambda$ of the order of a molecular size, $\delta_{\text {water }} \sim 0.3 \mathrm{~nm}$. This distance corresponds also to the average distance of minimal approach between proton-water and $\mathrm{Mn}^{2+}$ ions. The translational correlation time $\tau_{m}$ is associated with individual water molecular jumps at pore surfaces. The surface residence time, $\tau_{s}\left(>>\tau_{m}\right)$, which is limited by the molecular desorption from the surface layer $\lambda$ controls how long the proton species $I$ and $S$ stay correlated at pore surfaces. The ratio $\tau_{s} / \tau_{m}$ thus represents the dynamical surface affinity or NMR wettability [14]. The best fit obtained with Eq. 1 is displayed in Fig. 2 with a NMR specific surface area $S_{p, N M R}=47 \mathrm{~m}^{2} / g$, $\tau_{m}=12.0 \mathrm{ps}$ and the frequency independent bulk value $R_{1, \text { bulk }}^{\text {water }} \approx 30 \mathrm{~s}^{-1}$. For taking into account the rather dispersed data at low frequency, we have varied the activation energy $5 \leq E_{S} \leq 7.5 \mathrm{kcal} / \mathrm{mol}$ associated to the activated residence times $\tau_{S}=\tau_{S 0} \exp \left(E_{S} / R T\right)$ at pore surfaces. At high frequency $\left(\omega_{I}>0.3 \mathrm{MHz}\right)$ the entire fitting curves merge to a single one characterized by a single time of residence $\tau_{s}=0.6 \mu s\left(\tau_{s}>>\tau_{m}\right)$ consistent with a water-wet situation (Fig. 2). An estimation of the translational diffusion coefficient of 
water at the mineral clay-like surface thus gives $D_{\text {surf }}=\delta_{\text {water }}^{2} /\left(4 \tau_{m}\right)=1.88 \times 10^{-5} \mathrm{~cm}^{2} / \mathrm{s}$ which is slightly

lower than what is expected for bulk water at $\mathrm{T}=25^{\circ} \mathrm{C}$.

\subsection{NMRD profile of oil confined in the microstructure of kerogen.}

Based on our previous proton NMRD data in 1D pores [15], the inverse squared-root behavior with a leveling-off at low frequency shown as a red line in Fig. 2 strongly supports a relaxation process induced by a quasi-1D-translational diffusion of proton-oil species in proximity of paramagnetic $\mathrm{Mn}^{2+}$ ions at surface of kerogen micropores with an exponential cut-off after a surface time of residence $\tau_{S}$. Here again, the dominant feature of this relaxation process is the time dependence of the probability of reencounters between moving protons $I$ and fixed paramagnetic spins $S$ evolving at long times as $P(\tau) \propto \exp (-$ $\left.\tau / \tau_{S}\right) / \sqrt{ }\left(\tau / \tau_{m}\right)$. This gives the behavior $R_{1} \propto 1 / \sqrt{ } \omega_{I}$ observed in Fig. 2 at high frequency. The measured fractal pore-size distribution gives a number of pores $N(R) \propto\left(R / R_{\max }\right)^{-D f}$ of size $R$ in the range $\left\{R_{\min } \sim 2.5\right.$ $\left.n m-R_{\max } \sim 630 \mathrm{~nm}\right\}$ with the fractal dimension $D_{f} \sim 2.3[7,8]$. Due to the divergence of such a distribution for small nanopores, one finds that the highest number of pores $N(R)$ is around $R^{\sim 2.5-3} \mathrm{~nm}$. The following relations proposed [15] for the longitudinal and transverse relaxation rates induced by a translational diffusion of a liquid confined in 1D cylindrical nanopores allows reproducing almost all the features shown in Fig. (2):

$$
\begin{aligned}
R_{1, \text { oil }}\left(\omega_{I}\right)=R_{1, \text { bulk }}^{\text {oil }}+ & \sqrt{2} /\left(15 \pi R \delta_{1 D, \text { oil }}^{2}\right) \sigma_{s} \rho_{\text {oil }} S_{p, N M R}\left(\gamma_{I} \gamma_{s} \hbar\right)^{2} S(S+1) \sqrt{\tau_{m} \tau_{s}} \\
& \times\left[\frac{3 \sqrt{1+\sqrt{1+\omega_{I}^{2} \tau_{s}^{2}}}}{\sqrt{1+\omega_{I}^{2} \tau_{s}^{2}}}+\frac{7 \sqrt{1+\sqrt{1+\omega_{S}^{2} \tau_{s}^{2}}}}{\sqrt{1+\omega_{s}^{2} \tau_{s}^{2}}}\right] . \\
R_{2, \text { oil }}\left(\omega_{I}\right)=R_{2, \text { oulk }}^{\text {oil }} & +2 \sqrt{2} /\left(15 \sqrt{2} \pi R \delta_{1 D, \text { oil }}^{2}\right) \sigma_{s} \rho_{\text {oil }} S_{p, N M R}\left(\gamma_{I} \gamma_{s} \hbar\right)^{2} S(S+1) \sqrt{\tau_{m} \tau_{s}} \\
\times & {\left[1+\frac{\sqrt{1+\sqrt{1+\omega_{I}^{2} \tau_{s}^{2}}}}{\sqrt{1+\omega_{I}^{2} \tau_{s}^{2}}}+\frac{13 \sqrt{2}}{8} \frac{\sqrt{1+\sqrt{1+\omega_{S}^{2} \tau_{s}^{2}}}}{\sqrt{1+\omega_{S}^{2} \tau_{s}^{2}}}\right] . }
\end{aligned}
$$


In Eqs. 3 and 4 , the oil density is $\rho_{\text {oil }}=0.85 \mathrm{~g} / \mathrm{cm}^{3}$ and $R \sim 3.0 \mathrm{~nm}$ is the pore radius of the largest

population of pores following the fractal distribution described above. $\delta_{l D, \text { oil }} \sim \delta_{\text {oil }} / 2$ is the distance of minimal approach between $I$ and $S$ spins where $\delta_{o i l}$ is the average size of hydrocarbon (octane). This distance is taken at half of the molecular size because the saturated oil has no ligand field to the paramagnetic source of relaxation. The continuous curves of Fig. 2 have been fitted with Eqs. 3 and 4, with $S_{p, N M R}=30 \mathrm{~m}^{2} / \mathrm{g}$ and $\tau_{m}=4.1 \mathrm{~ns}$ for different values of the activation energy $6.6 \leq E_{S} \leq 7.4 \mathrm{kcal} / \mathrm{mol}$ associated to the activated time of residence $\tau_{S}=\tau_{S 0} \exp \left(E_{S} / R T\right)$ for considering the rather dispersed data at low frequency. We see that such a distribution of $E_{S}$ only affects the NMRD profile $1 / T_{1}\left(\omega_{I}\right)$ below 0.3 MHz. In the high frequency range where all the fits merge to a single theoretical profile $1 / T_{l}\left(\omega_{I}\right) \propto$ $1 / \sqrt{ } \omega_{I}$, we find a single surface residence time $\tau_{s}^{\sim} 0.78 \mu$ s. An estimation of the translational diffusion coefficient of oil at the surface of kerogen pores thus gives $D_{\text {surf }}=\delta_{\text {oil }}^{2} /\left(4 \tau_{m}\right)=2.58 \times 10^{-7} \mathrm{~cm}^{2} / \mathrm{s}$. We have introduced previously a dynamical surface affinity index $A=\tau_{s} / \tau_{m}$ that qualifies an average number of molecular steps on the pore surface giving a kind of local NMR wettability [14]. Here, we find $A \sim 195$ which is typical of an oil-wet situation in kerogen pores. The very small value of $D_{\text {surf }}$ compared to the bulk octane is due to the oil-wet condition favouring the dynamical surface affinity of the highly confined oil in kerogen nanopores.

\section{Experimental results, data treatment and discussion on $2 D N M R T_{1}-T_{2}$ and $T_{1} / T_{2}$ in shales}

The confined oil population has elongated peaks in Fig. 3a, b which exhibits a surprisingly high $T_{1} / T_{2}$ ratio around 10 at $2.5 \mathrm{MHz}$ and around $40-50$ at $23 \mathrm{MHz}$. On the contrary, the water peak stays fixed around $T_{1} / T_{2} \sim 1.4$. The high $T_{1} / T_{2}$ ratio could reveal viscous bitumen [1]. This hypothesis should be discarded due to the high ratio around 4-5 in presence of light oil [11]. Moreover, we did not observe by ESR the typical paramagnetic impurities of bitumen. In Figs. 3, we do not observe any cross peaks, thus proving the absence of proton exchange between oil and water populations on the time scale of 2D NMR 
1D pore-geometries, respectively:

$$
\begin{aligned}
& \frac{T_{1, \text { water }}^{2 D}}{T_{2, \text { water }}^{2 D}} \approx 2 \frac{\left[2 \operatorname{Ln}\left(\frac{\tau_{S}}{\tau_{m}}\right)+\frac{3}{4} \ln \left(\frac{1+\omega_{I}^{2} \tau_{m}^{2}}{\left(\tau_{m} / \tau_{s}\right)^{2}+\omega_{I}^{2} \tau_{m}^{2}}\right)+\frac{13}{4} \ln \left(\frac{1+\omega_{S}^{2} \tau_{m}^{2}}{\left(\tau_{m} / \tau_{s}\right)^{2}+\omega_{s}^{2} \tau_{m}^{2}}\right)\right]}{\left[3 \ln \left(\frac{1+\omega_{I}^{2} \tau_{m}^{2}}{\left(\tau_{m} / \tau_{s}\right)^{2}+\omega_{I}^{2} \tau_{m}^{2}}\right)+7 \ln \left(\frac{1+\omega_{s}^{2} \tau_{m}^{2}}{\left(\tau_{m} / \tau_{s}\right)^{2}+\omega_{s}^{2} \tau_{m}^{2}}\right)\right]}, \\
& \frac{T_{1, \text { oil }}^{1 D}}{T_{2, \text { oil }}^{1 D}} \approx 2 \sqrt{2} \frac{1+(3 \sqrt{2} / 8) \sqrt{1+\sqrt{1+\omega_{I}^{2} \tau_{s}^{2}}} / \sqrt{1+\omega_{I}^{2} \tau_{s}^{2}}+(13 \sqrt{2} / 8) \sqrt{1+\sqrt{1+\omega_{s}^{2} \tau_{s}^{2}}} / \sqrt{1+\omega_{s}^{2} \tau_{s}^{2}}}{3 \sqrt{1+\sqrt{1+\omega_{I}^{2} \tau_{s}^{2}}} / \sqrt{1+\omega_{I}^{2} \tau_{s}^{2}}+7 \sqrt{1+\sqrt{1+\omega_{s}^{2} \tau_{s}^{2}}} / \sqrt{1+\omega_{s}^{2} \tau_{s}^{2}}} .
\end{aligned}
$$

We remind that the 1D diffusion relaxation model presented in Section 3.2 for oil supposes a very long surface residence time $\tau_{s}\left(\gg>\tau_{m}\right)$ [5]. For the observed oil data, it appears from Eqs. 3 and 4 that $T_{1, \text { oil }}^{1 D} \propto \sqrt{\omega_{I} / \tau_{m}}$ independently of $\tau_{S}$ when $1 \leq \tau_{s} \leq 10 \mu \mathrm{s}$, while $T_{2}$ behaves as $T_{2, \text { oil }}^{1 D} \propto 1 / \sqrt{\tau_{m} \tau_{S}}$ for all $\tau_{s}$. This gives a ratio that behaves as $T_{1, \text { oil }}^{1 D} / T_{2, \text { oil }}^{1 D} \rightarrow 1$ when $\omega_{\mathrm{I}} \rightarrow 0$ and $T_{1, \text { oil }}^{1 D} / T_{2, \text { oil }}^{1 D} \propto \sqrt{\omega_{\mathrm{I}} \tau_{S}}$ at high frequency while $T_{2, \text { water }}^{2 D} / T_{2, \text { water }}^{2 D} \approx 1.4$ for water. We have displayed in Fig. 4a the frequency dependencies of $T_{1} / T_{2}$ for water (Eq. 5) and oil (Eq. 6) when varying $\tau_{s}$ in the range between $1 \mu s$ and $10 \mu s$ considering the large variety of oil surface dynamics in the kerogen pores. One notes that this ratio for oil could reach the values around $4-10$ at $2.5 \mathrm{MHz}$ and $40-50$ at $23 \mathrm{MHz}$. For water, the ratio $T_{2, \text { water }}^{2 D} / T_{2, \text { water }}^{2 D} \approx 1.4$ does not vary much in frequency (or in $\tau_{s}$ ) due to the 2D diffusive dimension and the small range of pore sizes.

Last, we have also displayed in Fig. $4 \mathrm{~b}$ the $2 \mathrm{D}$ spin-correlation map $T_{1}-T_{2}$ calculated at 2.5 and 23 $\mathrm{MHz}$ with Eqs. 1-4 by varying $\tau_{m}$ within $\{0.1-10 \mathrm{~ns}\}$ and $\{10-100 \mathrm{ps}\}$ for oil and water, respectively. We note that Fig. $4 \mathrm{~b}$ reproduces the two following relaxation features. (i) The oil peak shifts as $T_{1, \text { oil }}^{1 D} \propto \sqrt{\omega_{\mathrm{I}} \tau_{S}} T_{2, \text { oil }}^{1 D}$ in the range of large $T_{1}$ and $T_{2}$ values when increasing the frequency in excellent agreement with the proposed 1D relaxation theory. We have represented schematically in Fig. $4 \mathrm{~b}$ the leveling off $T_{1, \text { oil }}^{1 D} \propto \sqrt{\omega_{I} / \tau_{m}}$ where $T_{1}$ appears to be independent of $T_{2}$ within the short $T_{1}$ and $T_{2}$ values. 
the central peak of the $T_{1}-T_{2}$ correlation map as well as the large value observed for $T_{1} / T_{2}$ (Fig. 4a) can thus be explained by the low dimensionality of the oil diffusion at the huge kerogen pore surface. This result is of particular importance because it shows that measuring $T_{1}-T_{2}$ at different frequencies can inform on the fluid typing in confinement.

We discuss finally how to generalize our observed NMRD profiles to other shale samples. The kerogen maturity can have a direct effect on the specific surface area $S_{p, N M R}$ of the kerogen because it will develop more and more sponge-like porosity. As $R_{1, \text { oil }} \propto S_{p, N M R}$, the NMRD profile will thus be linearly dependent on the kerogen maturity. The value $S_{p, N M R}=30 \mathrm{~m}^{2} / \mathrm{g}$ used in our study is typical of a mature sample compared to a much lower value found for a weak kerogen maturity. Varying the nature (spin value $S$ ) and quantity (surface density $\sigma_{S}$ ) of paramagnetic sources of relaxation will only affect the absolute value of $R_{1, \text { oil }}$ but not its frequency dependence. The distribution of times of residence $\tau_{S}$ for oil at pore surfaces (Fig. 2) at low frequency can be universally encountered in other shale samples. Even, if the NMRD data are spatially limited to a few nanopores, the evidence of such a distribution at long times is consistent with the recent simulations and theoretical calculations proposed by several authors for interpreting the difficulty of expelling the petroleum fluids from the kerogen microstructure [16].

\section{Conclusion}

We have shown that fast-field-cycling NMR relaxometry allows probing non-invasively surface diffusion coefficients, residence times and local wettability of oil and water (brine) embedded in shale rocks. The high $T_{1} / T_{2}$ values found for oil are shown to be due to a lower diffusion dimensionality ( 1D) than for water (2D) and no bitumen presence has been required. Last, we have shown that measuring the 2D spin-correlation spectra $T_{1}-T_{2}$ at two frequencies gives confidence on fluid typing in the high confinement of shales. We believe that the frequency dependencies of the NMRD profiles which depend drastically on the pore geometry and local dimensionality give unambiguous information on the local dynamics of the embedded petroleum fluids. 
[1] P.M. Singer, E. Rylander, T. Jiang, R. McLin, R.E. Lewis, S.M. Sinclair, Society of Core Analysts, SCA2013-018.

[2] A.E. Ozen, R.F. Sigal, Petrophysics, 54 (2013) 11-19.

[3] E. Odusina, C. Sondergeld, C. Rai, Society of Petroleum Engineers, (2011) paper SPE147371.

[4] M. D. Hürlimann, L. Venkataramanan, J. Magn. Reson., 157 (2002) 31-42.

[5] J.-P. Korb, B. Nicot, A. Louis-Joseph, S. Bubicci, G. Ferrante, J. Phys. Chem. C., 118 (2014), 23212-23218.

[6] R. Kimmich, E. Anoardo, Prog. Nucl. Magn. Reson. Spectrosc, 44 (2004) 257-320.

[7] M.E. Curtiss, R.J Ambrose, C.H. Sondergeld, Ch. Rai, http://www.ogs.ou.edu/MEETINGS/Presentations/ShalesMoving2011/CurtisMicro.pdf (2011).

[8] M.E. Curtiss, R.J. Ambrose, Ch. Rai, CUSG/SPE (2010), 137693.

[9] J.-P. Butler, J.A., Reeds, , S.V. Dawson, SIAM J. Numer. Anal., 18 (1981) 381-397.

[10] L. Venkataramanan, Y.Q. Song, M.D. Hürlimann, IEEE Trans. Signal Proc., 50 (2002) 1017-1026.

[11]B. Nicot, N. Vorapalawut, B. Rousseau; L.F. Madariaga, G. Hamon, J.-P. Korb, Petrophysics, 15 (2015) 19-29.

[12]F. Barberon, J.-P. Korb, D. Petit, V. Morin, E. Bermejo, Phys. Rev. Lett., 90, (2003) 1-4.

[13]J.-P. Korb, M. Whaley-Hodges, R.G. Bryant, Phys. Rev. E, 56, (1997) 1934-1944.

[14] J.-P. Korb, G. Freiman, B. Nicot, P. Ligneul, Phys. Rev. E, 80 (2009) 061601-061612.

[15]F. Dalas, J.-P. Korb, S. Pourchet, A. Nonat, D. Rinaldi, J. Phys. Chem. C, 118 (2014) 8387-8396.

[16] Th. Lee, L. Bocquet, B. Coasne, Nature comm., DOI; 10.1038/ncomms (2016) 11890. 
Fig. 1 (a) FIB SEM micrographs of the microporosity of a shale oil rock sample.

Fig. 2 Logarithmic plot of the measured proton spin-lattice relaxation rate constants $R_{l}$ as a function of the proton Larmor frequency for oil/water/air shale. The continuous blue (water) and red (oil) lines are the best fits obtained with Eqs. 1 and 3, with the values obtained for $\tau_{m}$ and $\tau_{s}$. We have varied the activation energy $E_{s}$ at low frequency. We show in inset some examples of the bimodal $\mathrm{T}_{1}$-distributions obtained at different frequencies.

Fig. 3 (a) Two-dimensional $T_{1}-T_{2}$ spin-correlation maps observed for water and oil on "as received" shale-oil rock measured at $2.5 \mathrm{MHz}$. (b) ibid $T_{1}-T_{2}$ data measured at $23 \mathrm{MHz}$. The dashed lines indicate the values of $T_{1} / T_{2}$ ratio.

Fig. 4 (a) Theoretical dependencies of the ratio $T_{1} / T_{2}$ with Larmor frequency for a 2D diffusion of water in lamellar clay mineral (Eq. 5) and a 1D diffusion of oil in kerogen pores (Eq. 6). The circular points are the experimental values observed at 2.5 and $23 \mathrm{MHz}$ for oil in kerogen. The different continuous lines correspond to different values of $0.1 \leq \tau_{S} \leq 1 \mu \mathrm{s}$. (b) Theoretical $2 D T_{1}-T_{2}$ spin-correlation maps calculated from Eqs. 1-4 at 2.5 and $23 \mathrm{MHz}$ by varying $\tau_{m}$ within $\{0.1-10 \mathrm{~ns}\}$ and $\{10-100 \mathrm{ps}\}$ for oil and water, respectively. We have represented schematically in dotted lines the leveling off $T_{1, \text { oil }}^{1 D} \propto \sqrt{\omega_{I} / \tau_{m}}$ where $T_{1}$ appears to be independent of $T_{2}$ for short $T_{1}$ and $T_{2}$ values. 


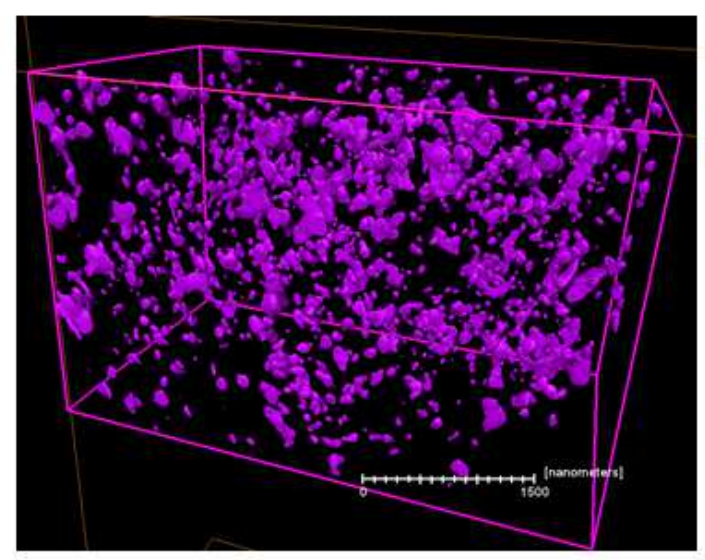

Fig. 1 


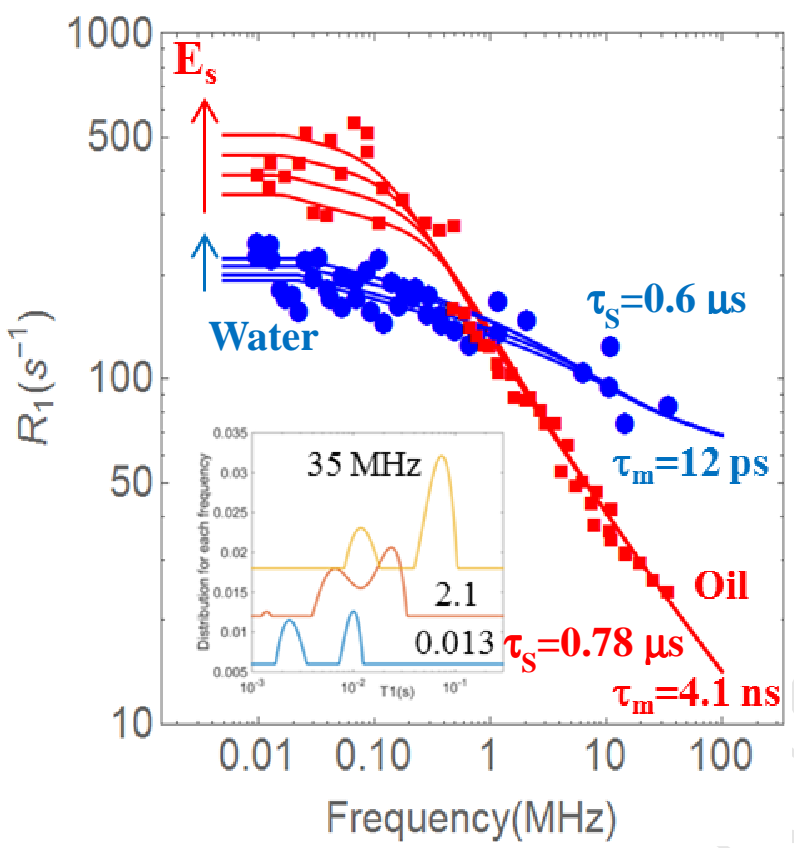

Fig. 2 

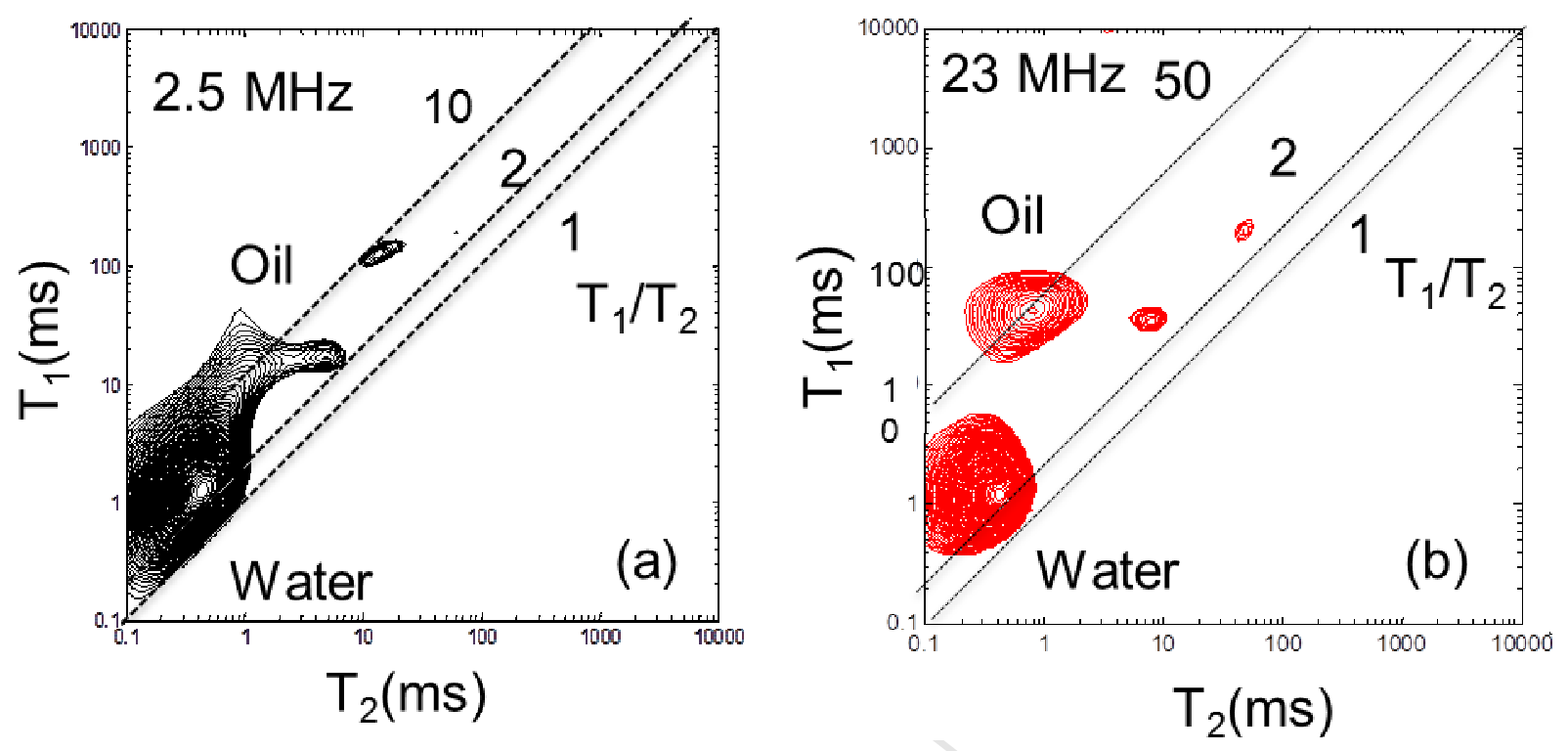

Fig. 3 

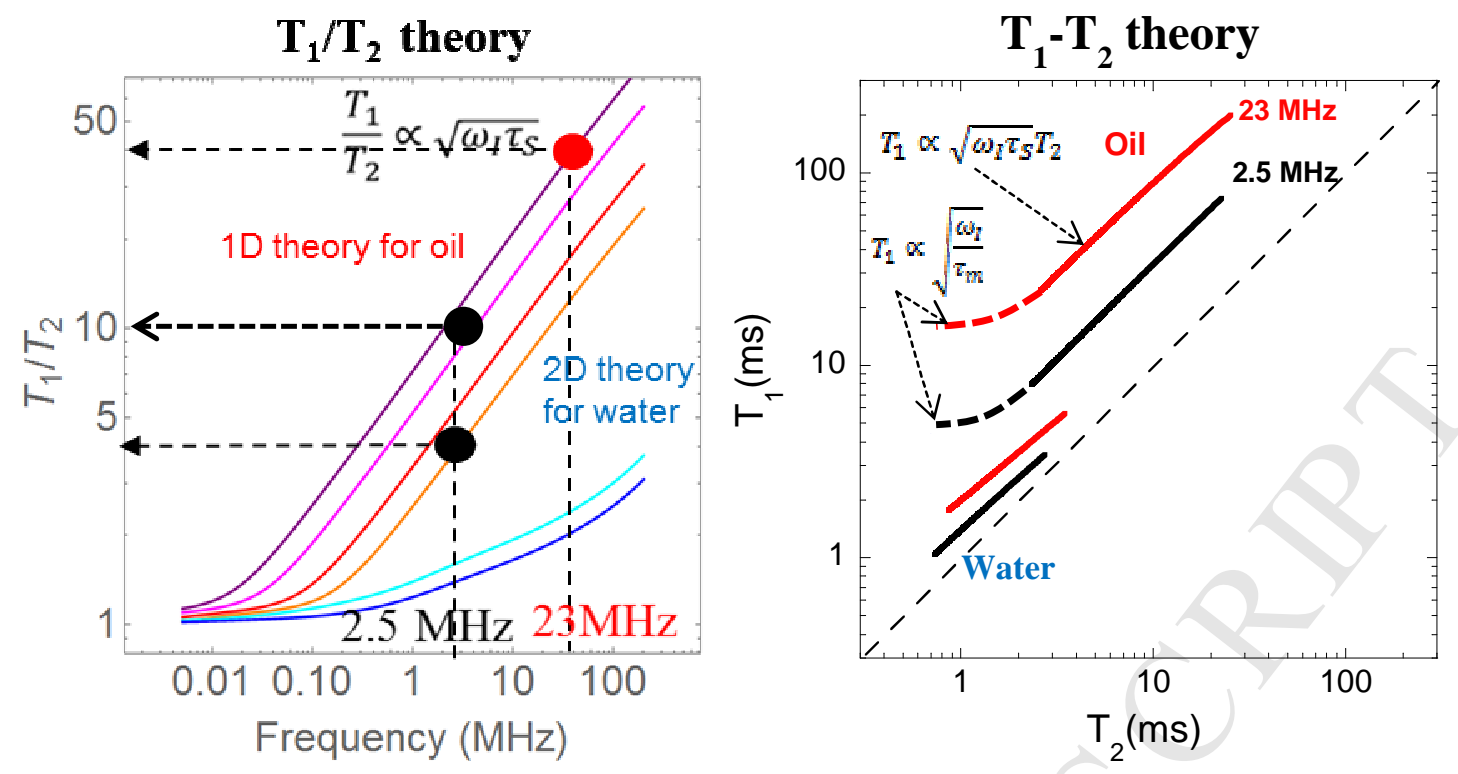

Fig. 4 


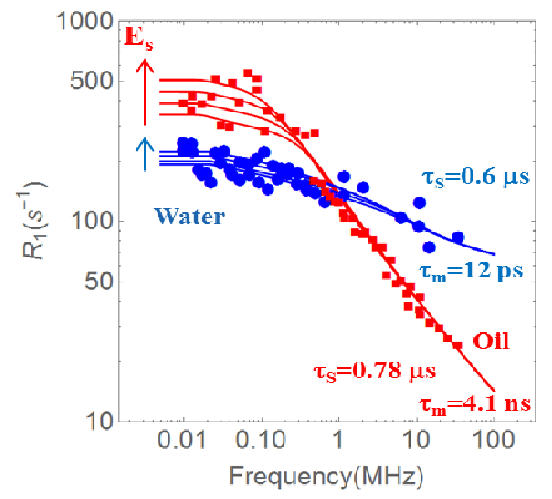




\section{Highligths}

- Nuclear magnetic relaxation dispersion profiles of longitudinal relaxation rates $1 / \mathrm{T}_{1}$ (NMRD) of oil and brine confined in shale oil rocks.

- Description of the nuclear relaxation models used for obtaining important dynamical and structural parameters from these experiments.

- New interpretation of the very different $T_{1} / T_{2}$ ratio observed for these petroleum fluids on the $2 \mathrm{D}$ spin-correlation maps $\mathrm{T}_{1}-\mathrm{T}_{2}$ observed at two frequencies.

- New information on the dynamics and wettability of the embedded fluids as well as some structural measurement on the kerogen pore size 